\title{
STUDY ON THE STABILITY OF STEINER TREE STRUCTURE OF EXPLOSION-PROOF TEXTILES
}

\author{
Ling-Ye $\mathrm{Wu}^{1}$, Ji-Huan $\mathrm{He}^{2,3}$ \\ ${ }^{1}$ College of Textiles, Donghua University, Shanghai, China \\ ${ }^{2}$ National Engineering Laboratory of Modern Silk, Soochow University, \\ No. 1, Shizi Street, Suzhou 215006, China \\ ${ }^{3}$ State Key Laboratory of Explosion Science and Technology, \\ Beijing Institute of Technology, Beijing 100081, China \\ Jhhe@dhu.edu.cn
}

\begin{abstract}
The stability of Steiner tree structure of explosion-proof textiles by studying on the tearing strength. We conclude that the Steiner tree structure has good stability.
\end{abstract}

Keywords- the Steiner tree structure, explosion-proof textiles, stability.

\section{INTRODUCTION}

In spite of great technological development and improvement of safety management, explosions in process equipment still claim lives and cause huge financial losses[1-3]. Although the most common incident in the process industry is fire, explosions cause the most severe losses. Grasping the characteristics of the explosion, we can, however, control it effectively using soft materials with Steiner tree structure[4]. An explosion is a phenomenon resulting from a sudden release of energy and instantaneous combination with high temperature and pressure, and it brings huge disaster in tens of seconds or even a few milliseconds. Therefore, the most effective control measure against explosion is prevention. So far, many experts and scholars have made outstanding contributions in this field (see [5-10]).

With the development of textile industry, the material of explosion-proof equipment changes from a rigid metal to a soft and high-performance fabric. However, the internal warp and weft structure of fabric materials is unstable under external forces. In fact, the net structure of fabric determines its performance. Thus we try to optimize textile structure to improve anti-explosion. It is a new idea to apply the mechanics of friction self-locking phenomenon and the classical Steiner tree theory in graph theory to design textile structure. Now we propose a new kind of explosion-proof textile with Steiner tree structure which can be used for civilian, military and other explosive-proof field.

\section{THE STABILITY OF STEINER TREE STRUCTURE}

In this section we illustrate the stability of Steiner tree structure of explosion-proof textiles. Figure 1 shows the topology structure of warp and weft of classical textiles, and the point $\mathrm{O}$ is its equilibrium position. If an external force from any direction is imposed on this net, it becomes instable immediately, and the net can not recover its original 
shape even when the external force disappears. Even worse, when there are two pieces of debris invading at the same time near the point $\mathrm{O}$, the net topology structure will be breakdown. Figure 2 shows another fabric with the Steiner tree structure, where $o_{1}$ and $o_{2}$ are equilibrium positions. When the net is subject to an external force from all directions, the force will be re-distributed among all points of the net fabric.

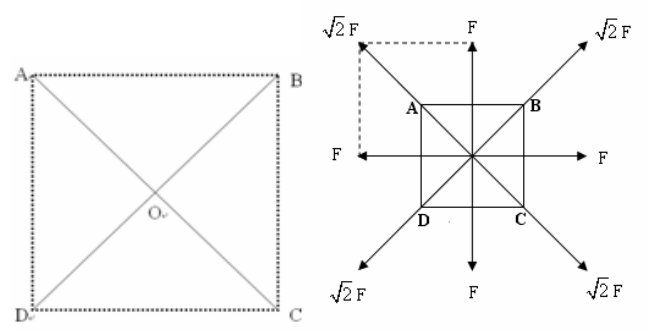

Fig. 1 The net structure of warp and weft

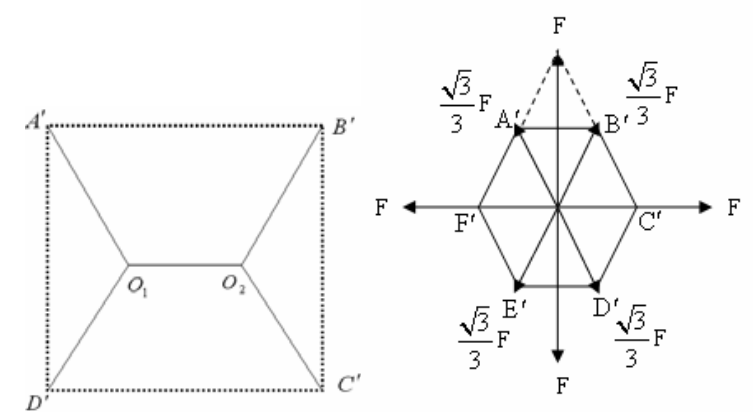

Fig. 2 The net structure with Steiner tree

When a force, F, is acted on the center of the net, each net point in Fig.1 is subject to an enlarged force, that is $\sqrt{2} F$; while that for Fig.2 is either $F / \sqrt{3}$ or F.

\section{EXPERIMENT}

\subsection{Experiment materials}

The sample shown in Table 1 is mesh fabric provided by Zhang Jia Gang Gang Hang Net Production Company.

Table 1 Sample standards

\begin{tabular}{|c|c|c|c|}
\hline width & $\begin{array}{c}\text { Component and } \\
\text { content }\end{array}$ & Mesh shape & weight \\
\hline $150 \mathrm{~cm}$ & Terylene100\% & sexangle & $50+/-5 \mathrm{~g} / \mathrm{m}^{2}$ \\
\hline
\end{tabular}

\subsection{Experimental method}

Experiment apparatus: Electronic Strength Testing Instrument YG065H .

Sample length: 200mm, sample width: $50 \mathrm{~mm}$.

Experiment method: single-rip tearing.

Gauge length: $100 \mathrm{~mm}$, velocity: $100 \mathrm{~mm} / \mathrm{min}$. 

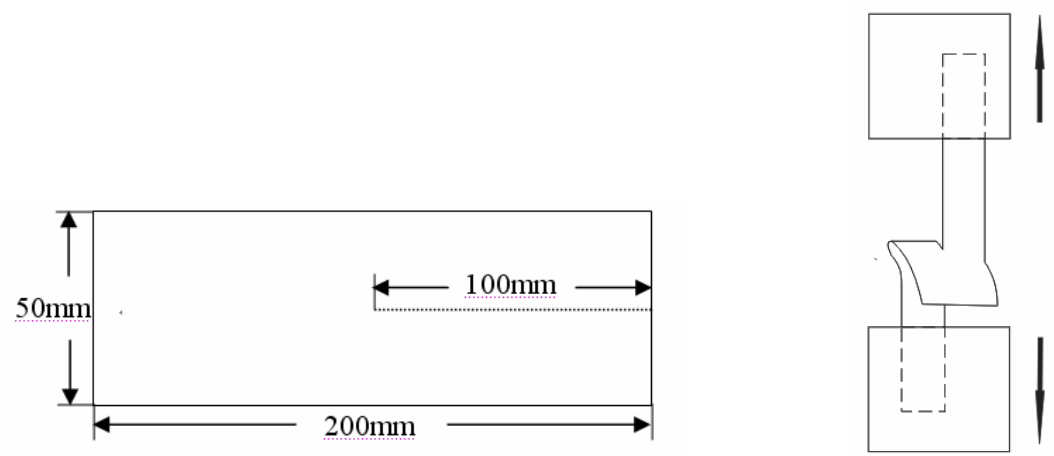

Fig. 3 The sample for the single-rip tearing

\subsection{Experiment results}

The sample's single-rip tearing strength is shown in Figure 4.

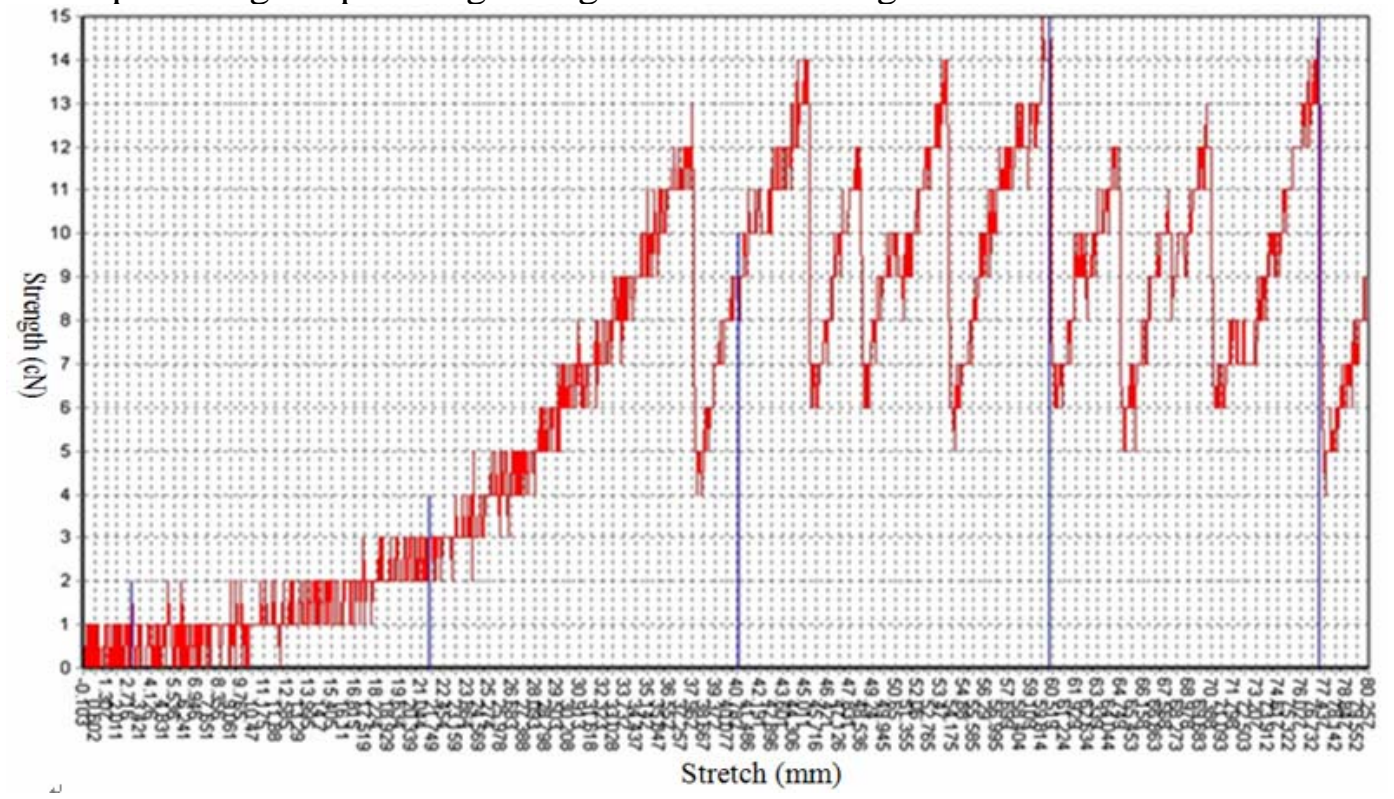

Fig. 4 Strength- stretch curve

\subsection{Discussion}

As shown in Figure 4, the net fabric with Steiner tree structure can absorb explosive energy effectively, even when points of the net are destroyed gradually, the net can still absorb explosive energy, though a sudden breaking of a net point leads to a sudden jump in force-resistance.

\section{CONCLUSION}

In this paper we have illustrated the stability of explosion-proof fabric with Steiner tree structure theoretically and experimentally. Any soft materials with Steiner tree structure are suitable for explosion-proof products. 


\section{ACKNOWLEDGEMENT}

The present work is supported by State Key Laboratory of Explosion Science and Technology, Beijing Institute of Technology under grant No. SKLEST-KF-09-01 (KFJJ09-01).

\section{REFERENCES}

1. J. H. He, L. F. Mo, Advances in Explosion Mechanics, Int. J. Nonlin. Sci. Num., 10, 977-978, 2009.

2. K. T. Wu, C. Wang, L. Zhang, et al. Moving Boundary Problem Arising in Explosion and Impact: An Algorithm for Youngs' Interface Reconstruction, Int. J. Nonlin. Sci. Num. 10, 1051-1057, 2009.

3. T. B. Ma, L. Ha, J. G. Ning, et al. Eulerian Numerical Method for Analysis of the Explosion and Impact Effects of Blast in Concrete, Int. J. Nonlin. Sci. Num. 10, 1005-1013, 2009.

4. J. H. He, Frontier of Modern Textile Engineering and Short Remarks on Some Topics in Physics, Int. J. Nonlin. Sci. Num. 11, 555-563, 2010.

5. F. D. Q. Alvarez, Explosion-proof device for measuring liquid levels, US Patent 4384184, 1983.

6. E. J. Falk, L. J. Brombolich, Explosion proof enclosure, US Patent 4664281, 1987.

7. D. D. Hakarine, Explosion-proof vent barrel for a battery, US Patent 4002495, 1977.

8. T. F. Roche, Explosion-proof emergency light, US Patent 4156891, 1979.

9. F. H. Slocum, Explosion proof junction box, US Patent 3724706, 1973.

10. R. Zalosh, New Developments in Explosion Protection Technology, Fire and Emergency Services Asia 2005, Singapore. 